\title{
A Robotic Assistance Machine Vision Technique for an Effective Inspection and Analysis
}

\author{
Santosh Kumar Sahoo*, B. B. Choudhury** \\ * Departement of Electronics \& Telecommunication Engineering, IGIT, Sarang, Utkal University, Odisha, India \\ **Department of Mechanical Engineering, IGIT, Sarang
}

\begin{tabular}{l} 
Article Info \\
\hline Article history: \\
Received Oct 19, 2014 \\
Revised Dec 17, 2014 \\
Accepted Jan 4, 2015
\end{tabular}

Keyword:

Industrial Robort

LABVIEW software

Smart Camera

Vision builder

\begin{abstract}
An Inspection is a study of methods and techniques that can be suitably employed in practical applications. In this paper, a new activity is proposed and analysis framework to facilitate the inspection of an object using machine vision technique in which maximum efficiency can be achieved. By using LABVIEW software and vision builder software the quality of output images such as image compression, image restoration and multimedia streaming are achieved successfully. So the proposed design makes use of various image processing functions like special filters and classifiers to compute the optimum results. Using smart camera in the inspection system the static as well as the dynamic object is captured in fraction of seconds without any blurs; as a result the optimum image quality without any distortion is obtained for better analysis. The proposed system is very precise, accurate and flexible with reasonable development cost compared to other model. With the aid of an Industrial robotic system with simulation software the object is replaced immediately when the same is rejected by the machine vision model. Apart from this, the proposed model can be implemented for any type of Automation work
\end{abstract}

Copyright (C) 2015 Institute of Advanced Engineering and Science. All rights reserved.

\section{Corresponding Author:}

Santosh Kumar Sahoo

Departement of Electronics \& Telecommunication Engineering

Igit, Sarang, Utkal University, Odisha, India

Email: santosh.kr.sahoo@gmail.com

\section{INTRODUCTION}

It is an obligatory step to evaluate the quality of output images in many image processing applications such as image acquisition, compression, restoration, transmission, etc. Since human beings are the ultimate observers of the processed images and thus the judges of image quality, it is highly desired to develop automatic approaches that can predict perceptual image quality consistently with human subjective evaluation.

Machine vision is a branch of engineering that uses computer vision in the context of manufacturing, where the images analysis is done to extract data for controlling a processor activity. Machine vision processes are targeted at recognizing the actual objects in an image and assigning properties to those objects-understanding what they mean. Engineers are adding Machine vision systems to a number of industrial applications to reduce costs, increase throughput, and improve customer satisfaction. All machine vision systems include a combination of hardware and software to acquire and process images, usually resulting in a response from a secondary system connected to the inspection system. There are two possible designs for this reference architecture. One includes a low-cost, rugged, embedded solution while the other makes use of the power of a computer to acquire and process images at a higher rate and resolution. Vision systems can be used to precisely measure number of variables such as length, angle, position, orientation, color and so on. The main advantage of a vision measuring system in these applications is its non-contact feature, which is important in cases when it is difficult to implement contact measurement. 
This paper proposed the devices and method to develop an effective inspection system. The system consists of Smart Camera 1722, a motion platform, Lab view Window and NI vision development module. In this proposed model the investigation of the circular linearity by using the NI Vision system which consist of the following Parts such as
[A] Illumination
[B] Object
[C] Smart Camera
[D] Image processor
[E] Controller (Robotic)

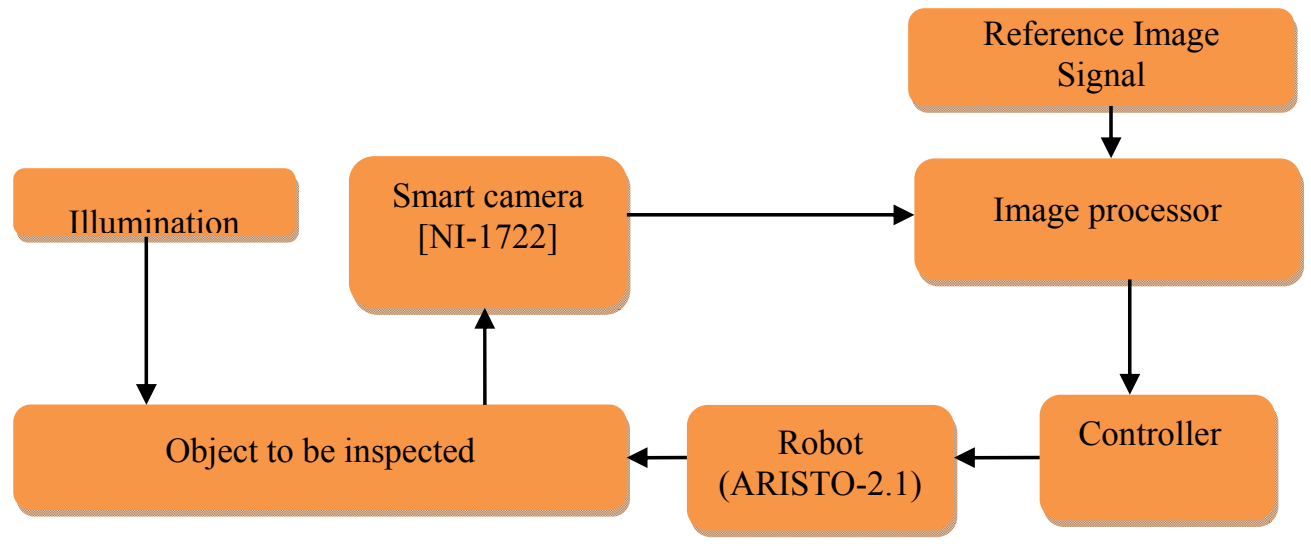

Figure 1. Schematic Block diagram of Object Inspection System

A. Illumination:

Lighting includes use of both artificial light sources such as lamps and natural illumination of interiors from daylight. Artificial lighting is most commonly provided today by electric lights. When the information from the samples is accumulated and analyzed, with respect to the specific sample and inspection requirements, we can achieve the primary goal of NI vision lighting analysis - to provide sample appropriate lighting that meets three acceptance criteria consistently:

* Maximize the contrast on those features of interest

* Minimize the contrast elsewhere

* Provide for a measure of robustness.

B. Object:

The part which is going to be inspected in terms of circular linearity. In this particular paper the cylindrical bar is placed for investigating its circular linearity.

C. Smart Camera 1722

The selection of camera is heavily dependent on our application. If select an appropriate camera, lens and lightning setup, the efforts can then be focused on developing our solution, rather than wrestling with poor image data and saves processing time at execution. Here NI smart camera1722 is selected and having resolution of $1280 \times 1024$ with $7.4 \times 7.4 \mu \mathrm{m}$ pixel size.

D. Image processor

It is used for processing the acquired image from the smart camera for results with actual predefined parameters.

E. Controller (Robotic):

The Robotic controller gives the command signal to the Robot after successful analysis by means of circularity matching with predefined parameters; as a result the defective objects can be replaced form the test bench. The Block diagram of Object Inspection System presented in Figure 1.

\section{ALGORITHM}

The algorithm comprises the various analyzing and processing functions illustrated below. 


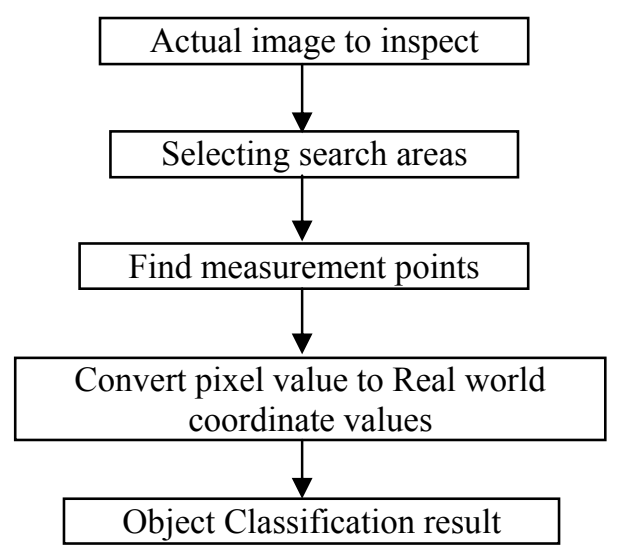

Figure 2. Algorithm chart for Inspection of an object

The image is captured by the NI SMART CAMERA (NI-1722). Vision Assistant offers three types of acquisitions: snap, grab, and sequence. A snap acquires and displays a single image. The grab acquires and displays images in a continuous mode at maximum rate, which is useful when you need to focus the camera. The sequence acquires images according to settings that specify in the Sequence tab of the Acquisition Interface window and sends the images to the Image Browser.

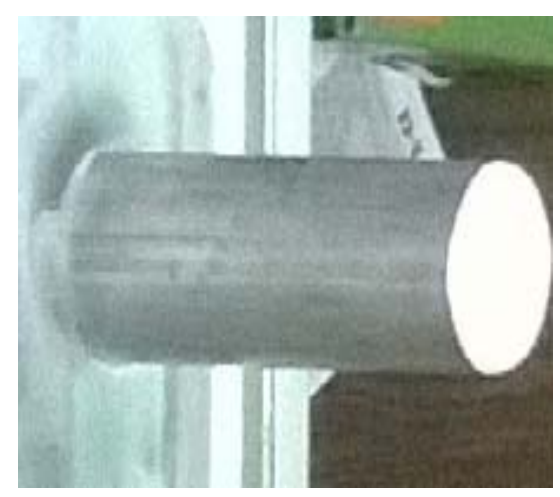

Figure 3. Actual image captured by Smart Camera

The origin image's size is $1360 \times 1024$, it is so big that it will waste long time to process. So it is necessary to resize the origin image by scale of 0.2 . But the result must multiply the same scale of 5 correspondingly to recover its real position in origin image.

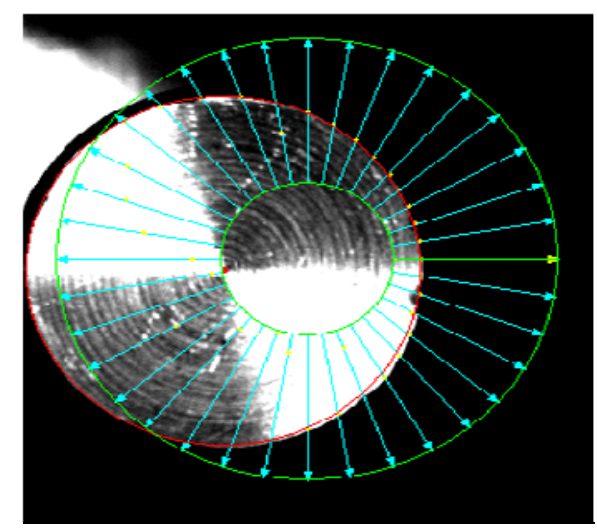

Figure 4. Selecting the desired mark points 
Classification identifies an unknown sample by comparing a set of its significant features to a set of features that conceptually represent classes of known samples. The object classifier uses feature vectors to identify samples based on their shape. Color classifier uses color features to identify samples based on their color. Classification involves two phases: training and classifying. Training is a phase during which we teach the machine vision software the types of samples want to classify during the classifying phase. It can train any number of samples to create a set of classes, which, later compare to unknown samples during the classifying phase and stored in the classes in a classifier file. Training might be a one-time process, or it might be an incremental process repeat to add new samples to existing classes or to create several classes, thus broadening the scope of samples to classify. The classifying phase classifies a sample according to how similar the sample features are to the same features of the trained samples. The need to classify is common in many machine vision applications. Typical applications involving classification include the following:

- Sorting

- Inspection

Here the geometric matching algorithm is used to detect the mark of circle. Geometric matching is an important tool for machine vision applications; it must work reliably under various, sometimes harsh, conditions. In automated machine vision applications - especially those incorporated into manufacturing process - the visual appearance of materials or components under inspection can change because of factors such as varying part orientation, scale, and lighting. The geometric matching tool must maintain its ability to locate the template patterns despite these changes.

The geometric matching process consists of two stages: learning and matching. During the learning stage, the geometric features from the template image. The algorithm organizes and stores these features and the spatial relationships between these features in a manner that facilitates faster searching in the inspection image. During the matching stage, the geometric matching algorithm extracts geometric feature from the inspection image that correspond to the features in the template image. Then, the algorithm finds matches by locating regions in the inspection image where features align themselves in spatial patterns similar to the spatial patterns of the features in the templates.

In Lab view Windows/CVI, the function imaq Detect Circles based geometric matching is used to perform the task. In the function, the threshold which specifies the minimum contrast a seed point must have in order to begin a curve must be set carefully. The inspection result is listed in Table I. The coordinate origin is in top left corner of the inspected image by default. There exits a little difference corresponding to different resize scale. The reason lies in the interpolation method when resize the origin image using Resample function in Lab View Windows. But it doesn't affect the inspection result if we convert the coordinate to the benchmark.

Table 1. Mark inspection result

\begin{tabular}{ccccccc}
\hline Scale & 0.6 & 0.5 & 0.4 & 0.35 & 0.3 & 0.2 \\
\hline X (pixel) & 883.33 & 880 & 882.5 & 887.14 & 833.33 & 815 \\
Y (pixel) & 685 & 688 & 652.5 & 652.86 & 656.67 & 660 \\
Time (ms) & 22.2 & 16.9 & 16.8 & 12.2 & 11.8 & 9.2 \\
\hline
\end{tabular}

\subsection{Coordinate Conversion}

Before inspection, the system must be able to relate regions of particular images to be inspected. However, in an inline inspection system, when an object moving into the inspection position, there are some minor positional or orientation errors because of the mechanical factor and the interpolation factor. To eliminate the deviation, it is necessary to align the object prior to inspection. This paper presents an algorithm to rectify the error from the above factor.

As shown in Figure 5, $\mathrm{P}$ and $\mathrm{Q}$ are marks in database with coordinate $(X \mathrm{p}, Y \mathrm{p})$ and $(X \mathrm{q}, Y \mathrm{q}), P^{\prime}$ and $Q^{\prime}$ are marks inspected from a sample object with coordinate $\left(X \mathrm{p}^{\prime}, Y \mathrm{p}^{\prime}\right)$ and $\left(X \mathrm{q}^{\prime}, Y \mathrm{q}^{\prime}\right)$. The midpoint of $P$ and $Q$ is $O(X \mathrm{o}, Y \mathrm{o})$, and the midpoint of $P^{\prime}$ and $Q^{\prime}$ is $\mathrm{O}^{\prime}\left(X \mathrm{o}^{\prime}, Y_{\mathrm{o}}{ }^{\prime}\right)$. Their coordinate can be calculated as the following equations. 


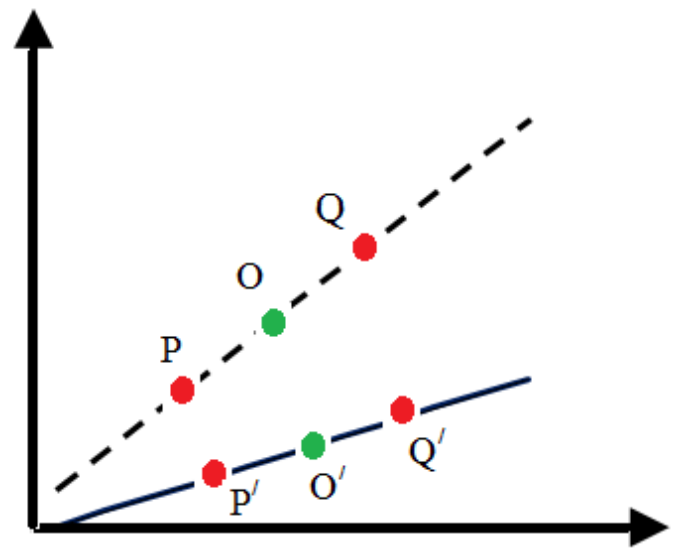

Figure.5: Marks in an in spected object and reference data in database.

$$
\begin{aligned}
& X_{O}=\frac{X_{p}+X_{q}}{2} \\
& Y_{O}=\frac{Y_{p}+Y_{q}}{2}
\end{aligned}
$$

According to above equation, we can convert the coordinate system of the real object in to the database coordinate system. A pixel point in an object with coordinate $\left(P^{\prime}, Q^{\prime}\right)$ can convert to database coordinate point $(P, Q)$ as following equation

$$
\mathrm{P}=P_{X} \mathrm{P}^{\prime}, \mathrm{Q}=P_{Y} \mathrm{Q}^{\prime}
$$

\subsection{Pattern Matching and Inspect the Wrong Parts}

Pattern matching quickly locates regions of a grayscale image that match a known reference pattern, also referred to as a model or template. When using pattern matching, first create a template that represents the objects for which you are searching. Pattern matching application then searches for instance of the template in each acquired image, calculating a score for each match. This score relates how closely the template resembles the located matches. Pattern matching finds template matches regardless of lighting variation, blur, noise, and geometric transformation such as shifting, rotation, or scaling of the template. NI pattern matching techniques include normalized cross-correlation, pyramidal matching, scale-and rotationinvariant matching, and image understanding. Normalized cross-correlation is the most common method for building a template in an image. The following is the basic concept of correlation: consider a sub image 
$\mathrm{R}(\mathrm{p}, \mathrm{q})$ of size $k \times l$ within an image $f(\mathrm{p}, \mathrm{q})$ of size $m \times m$, where $k \leq m$ and $l \leq n$. The correlation between $\mathrm{R}(\mathrm{p}, \mathrm{q})$ and $f(p, q)$ at point $(i, j)$ is given by

$$
C(i, j)=\sum_{p=0}^{l-1} \sum_{q=0}^{k-1} R(p, q) f(p+i, q+j)
$$

Where $i=0,1 \ldots m-1, j=0,1, \ldots n-1$, and the summation is taken over the region in the image where $R$ and $f$ overlap. Basic correlation is very sensitive to amplitude changes in the image, such as intensity, and in the template.

For example, if the intensity of the image $f$ is doubled. So are the values of $C$. We can overcome sensitivity by computing the normalized correlation coefficient, which is defined as

$$
\begin{aligned}
& S(i, j)=\frac{\sigma(i, j)}{\sqrt{D(R)} \sqrt{D[f(i, j)]}} \\
& \sigma(i, j)=\sum_{p=0}^{l-1} \sum_{q=0}^{k-1}(R(p, q)-\bar{R})(f(p+i, q+j)-\bar{f}(i, j) \\
& D(R)=\sum_{p=0}^{l-1} \sum_{q=0}^{k-1}(R(p, q)-\bar{R}) \\
& D[f(i, j)]=\sum_{p=0}^{l-1} \sum_{q=0}^{k-1}(f(p+i, q+j)-f(i, j))^{2}
\end{aligned}
$$

where $\bar{R}$ (calculated only once) is the average intensity value of the pixels in the template $R$. The variable $f$ is the average value of $\mathrm{f}$ in the region coincident with the current location of $R$. The value of $S(i, j)$ lies in the range -1 to 1 and is independent of scale changes in the intensity values of $f$ and $R$. Normalized cross-correlation is a good technique for finding patterns in an image when the patterns in the image are not scaled or rotated. Typically, cross-correlation can detect patterns of the same size up to a rotation of $5^{\circ}$ to $10^{\circ}$. Scale invariant matching adds a significant amount of computation to matching process. To resolve the problem, NI improves the computation time of pattern matching by reducing the size of the image and the template by pyramidal matching. In pyramidal matching, both the image and the template are sampled to smaller spatial resolutions. Because the image is smaller, matching is faster. When matching is complete, only areas with high match scores need to be considered as matching areas in the original image.

\section{RESULTS AND ANALYSIS}

We performed the proposed algorithm and method with Lab View Windows/CVI to detect the quality of an circular object. Figures 6-8 are the software user interface which are designed to detect the different edge position. All the aforesaid techniques are followed by using the NI vision in which the investigation of the circularity of an object as follows 


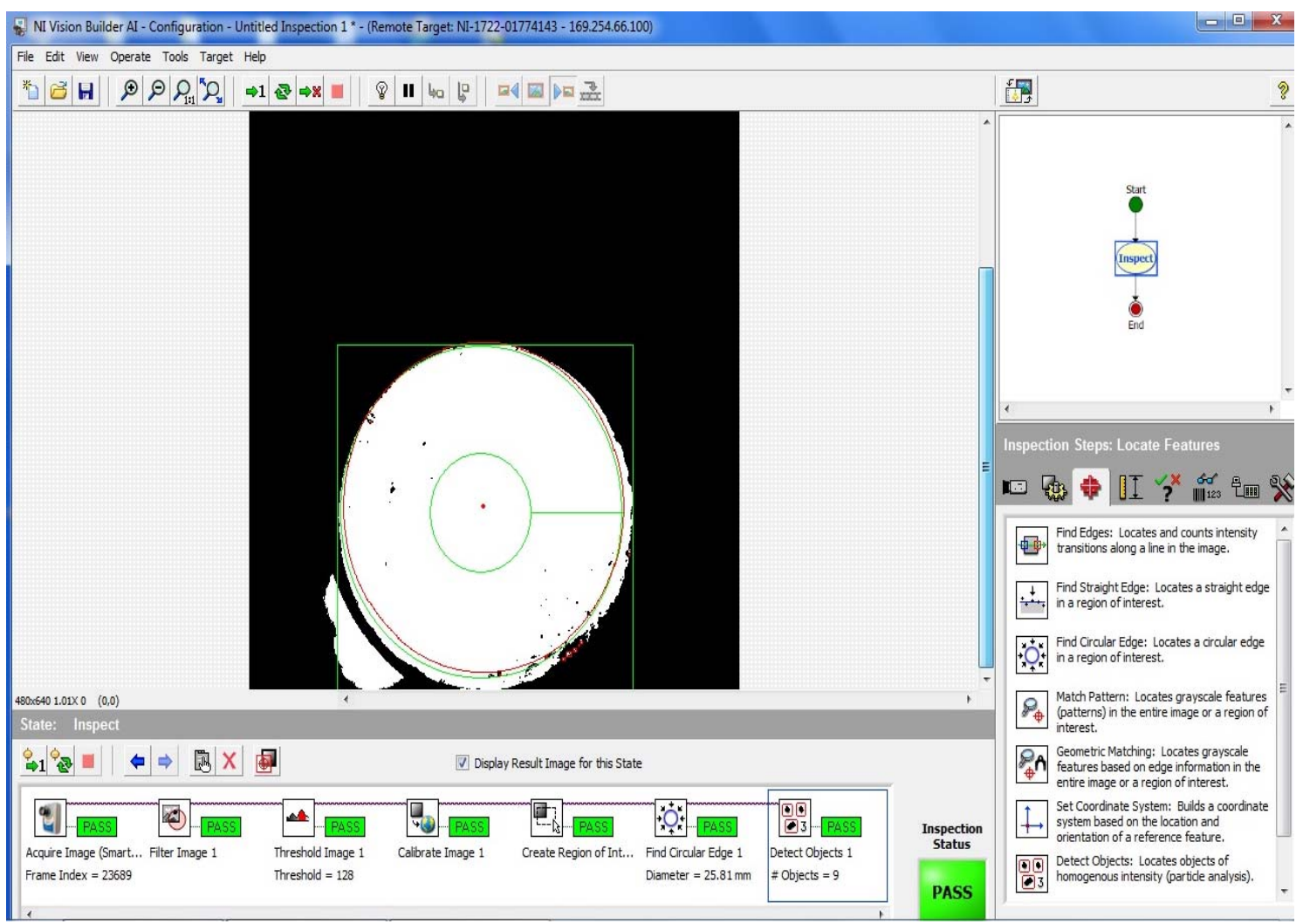

Figure 6. Simulated object by the software

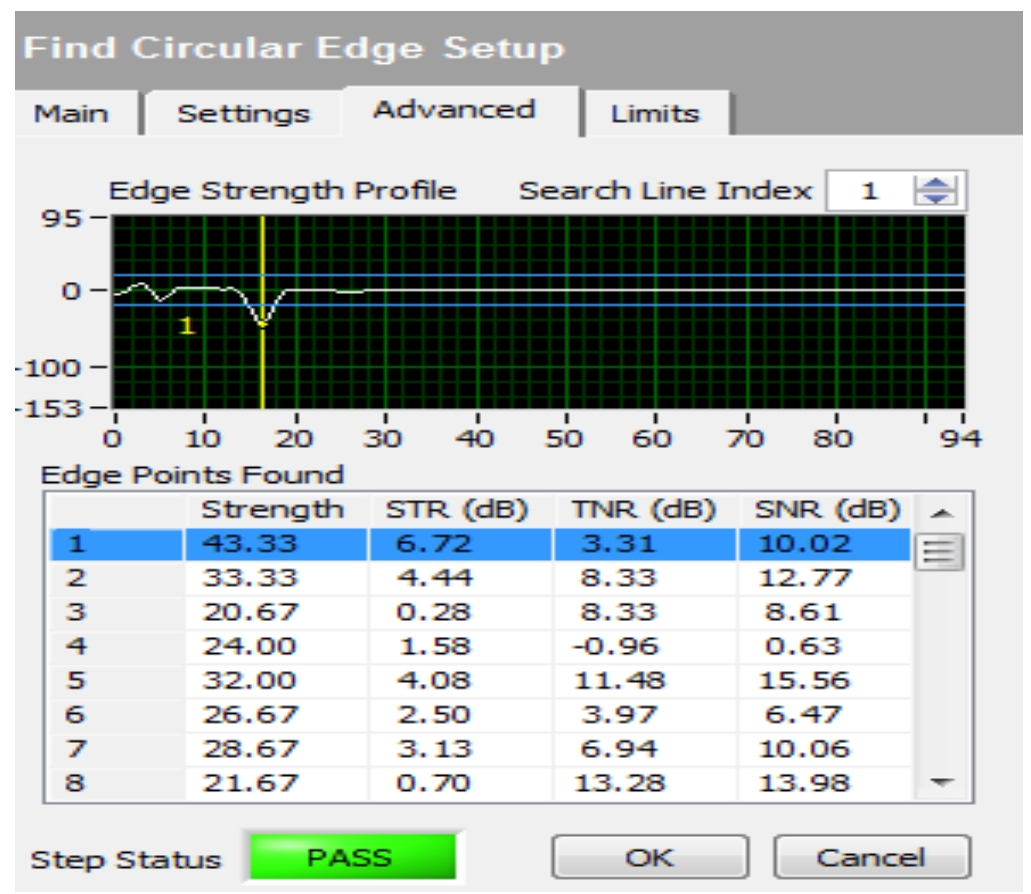

Figure 7. Circular edge parameters setting points 


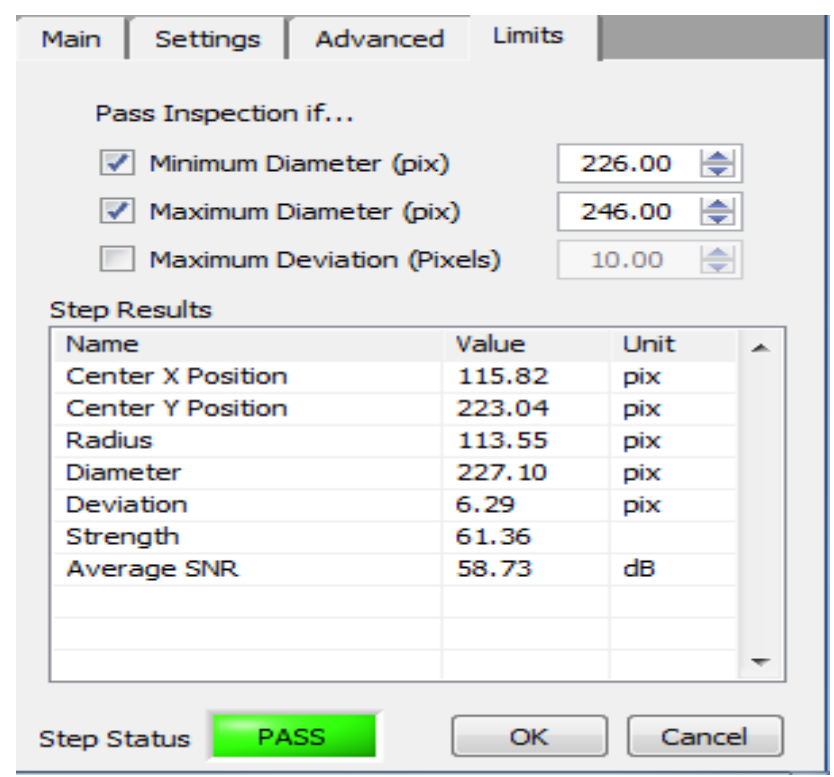

Figure 8. Circular edge parameters setting points

\section{CONCLUSION}

The computational speed of image processing has been improved using Machine Vision builder. A new system based on machine vision helps to calculate the results with precise and accurate values. Object Classification is an effective technique to classify or inspect the Circular linearity automatically and give results to match with the previously saved template. Filters help to improve the quality of an image. Image quality is basically depends upon the illumination conditions which produces noise in the image.

\section{REFERENCES}

[1] Z. Wang, et al., "Modern Image Quality Assessment", Synthesis Lectures on Image, Video, and Multimedia Processing, vol. 2, no.1, pp. 1-156, 2006.

[2] Richard P. et al., "A machine-vision system for iris recognition David Samoff Research Center, Inc", Princeton, NJ 08543-5300, USA ,1996, Page No: 1-8

[3] Franci Lhajnar, et al., "Machine Vision system for inspecting electric plates", Elsevier, computer in industry, 47(2002), Page No: 113-122.

[4] Joze Derganc, et al., "A machine vision system for measuring the eccentricity of bearings", Elsevier, page no.103111.

[5] Tadhg, Brosnan, et al. "Improving quality inspection of food products by computer vision-a review", Journal of Food Engineering, 61, (2004), Page No: 3-16.

[6] T. S. White ,et al., : "A Mobile Climbing Robot for High Precision Manufacture and Inspection of Aero-structures", The International Journal of Robotics Research, 2005; DOI: 10.1177/0278364905055701, Page No:- 589-598,

[7] Se-gon Roh et al., "Differential-Drive In-Pipe Robot for Moving Inside Urban Gas Pipelines", IEEE transactions on robotics, vol. 21, no. 1, February 2005, Page No:1-17.

[8] Andr'e Treptow, et al., "Real-time people tracking for mobile robots using thermal vision", Elsevier: Robotics and Autonomous Systems, 54 (2006), Page No: 729-739.

[9] Marko Heikkila et al., "A Texture-Based Method for Modeling the Background and Detecting Moving Objects", IEEE transactions on pattern analysis and machine intelligence, vol. 28, no. 4, April 2006, Page No: 657-662.

[10] Yakov Frayman, et al., "Machine Vision System for Automatic Inspection of Surface Defects in Aluminum Die Casting", Journal of Advanced Computational Intelligence and Intelligent Informatics, Vol.10 No.3, 2006, Page No281-290. 


\section{BIOGRAPHIES OF AUTHORS}

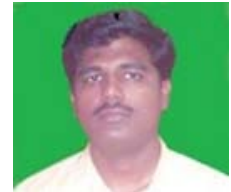

S.K. Sahoo, obtained his M.Tech in Electronics and Instrumentation Engineering. He is currently a Ph.D Scholar, Dept. of Electronics \& Communication Engineering at Utkal University, Bhubaneswar, Odisha, India Mr. Sahoo is a member of ISTE.IE and IEEE

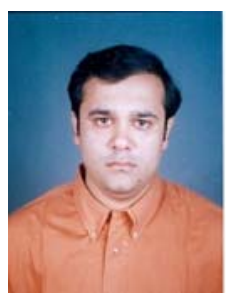

B.B. Choudhury, obtained his Ph. D. in Task Allocation strategies in Multi - Robot Environment. He is currently a Assistant Professor at I.G.I.T, Sarang in the Department of Mechanical Engineering. His current research interest includes Robotics, FMS, CAD/CAM/CIM, and Soft computing. Dr. Choudhury is a member of ISTE, IE(I), IACSIT,IAENG. 